\title{
Large paradoxical embolus through a patent foramen ovale following arteriovenous graft thrombectomy
}

\author{
Chadi Allam ${ }^{1}$, Zeina Kadri ${ }^{1}$, and Rabih Azar ${ }^{1}$ \\ ${ }^{1}$ Hotel-Dieu De France
}

September 24, 2021

\begin{abstract}
An 86-year-old man with end-stage renal disease on hemodialysis with an arteriovenous fistula in his left upper extremity presented to his hemodialysis session with thrombosis of his arteriovenous fistula. The patient underwent surgical thrombectomy. The patient later showed evidence of peripheral embolization and livedo reticularis. Transthoracic and transesophageal echocardiograms revealed a large thrombus $(5 \times 2 \mathrm{~cm})$ in the left atrium prolapsing to the right atrium via a patent foramen ovale and another thrombus (white arrow) adherent to the apical wall of the right ventricle. The thrombus in the left atrium was intermittently crossing the mitral valve and entering the left ventricle.
\end{abstract}

Large paradoxical embolus through a patent foramen ovale following arteriovenous graft thrombectomy

Running head: Large thrombus through a patent foramen ovale

Chadi Allam ${ }^{1,2}$, Zeina $\operatorname{Kadri}^{1,2}$, Rabih Azar ${ }^{1,2}$

${ }^{1}$ Faculty of Medicine, Saint-Joseph University, Beirut, Lebanon.

${ }^{2}$ Division of Cardiology, Hôtel-Dieu de France Hospital, Beirut, Lebanon.

First author: Chadi Allam, MD

Corresponding author: Rabih Azar, MD, MPH, FACC

Division of Cardiology, Hôtel-Dieu de France Hospital, Alfred Naccache Blvd, Beirut, Lebanon.

Tel: +9613590999

Fax: none

Email: razar@usj.edu.lb

Chadi Allam: chadiallammd@gmail.com

Zeina Kadri: zkadri@hotmail.com

Key words: echocardiography, ultrasound, thrombosis, paradoxical embolism, patent foramen ovale

Conflict of interest: none

Abstract

An 86-year-old man with end-stage renal disease on hemodialysis with an arteriovenous fistula in his left upper extremity presented to his hemodialysis session with thrombosis of his arteriovenous fistula. The patient underwent surgical thrombectomy. The patient later showed evidence of peripheral embolization and livedo reticularis. Transthoracic and transesophageal echocardiograms revealed a large thrombus $(5 \times 2 \mathrm{~cm})$ 
in the left atrium prolapsing to the right atrium via a patent foramen ovale and another thrombus (white arrow) adherent to the apical wall of the right ventricle. The thrombus in the left atrium was intermittently crossing the mitral valve and entering the left ventricle.

An 86-year-old man with end-stage renal disease on hemodialysis with an arteriovenous (AV) graft in his left upper extremity presented to his hemodialysis session with orthostatic hypotension and orthopnea. Clinical examination revealed thrombosis of his AV fistula. A transthoracic echocardiogram (TTE) showed global hypokinesia of the left ventricle (LV) with an estimated ejection fraction of $25 \%$ and no significant valvular disease. The patient underwent surgical thrombectomy and was admitted to the intensive care unit (ICU), where he underwent hemodialysis. We initiated anticoagulation with unfractionated heparin.

Following his admission to the ICU, the patient showed evidence of peripheral embolization and livedo reticularis. Ultrasound revealed occlusion of the left tibial artery. A chest CT-angiogram ruled out pulmonary embolism. Brain MRI showed multiple small infarcts. A second TTE and a transesophageal echocardiogram revealed a large thrombus (red arrow) $(5 \times 2 \mathrm{~cm})$ in the left atrium (LA) prolapsing to the right atrium (RA) via a patent foramen ovale (FO) and another thrombus (white arrow) adherent to the apical wall of the right ventricle (RV) (Figure panels A, B and C, Videos 1 and 2). The thrombus in the LA was intermittently crossing the mitral valve and entering the LV (Figure panel A, Video 1). Due to high surgical risk, we adopted a noninvasive approach consisting of anticoagulation with unfractionated heparin.

Follow-up TTE three weeks later showed a decrease in thrombus size (Figure panels D and E, Videos 3 and $4)$.

This is a rare documentation of a large thrombus crossing a patent foramen ovale and causing paradoxical embolization.

Figure legend:

A, Baseline echocardiography, apical four chamber view, showing a large thrombus in the left atrium crossing the mitral valve and entering the left ventricle and also prolapsing to the right atrium via the foramen ovale (red arrows). Another thrombus appears adherent to the apical wall of the right ventricle (white arrow). B and C, Transesophageal echocardiogram with 3D reconstruction showing a thrombus (red arrows) in the left atrium crossing the foramen ovale and prolapsing in the right atrium. D and E, Follow-up transthoracic echocardiography three weeks later showing a decrease in thrombus size (red arrows). LV = left ventricle; $\mathrm{RV}=$ right ventricle; $\mathrm{LA}=$ left atrium $; \mathrm{RA}=$ right atrium; $\mathrm{FO}=$ foramen ovale.

Video 1: Transthoracic echocardiography apical four chamber view showing a large thrombus in the left atrium crossing the mitral valve and entering the left ventricle and also prolapsing to the right atrium via the foramen ovale. Another thrombus appears adherent to the apical wall of the right ventricle.

Video 2: Transesophageal echocardiogram showing a thrombus in the left atrium crossing the foramen ovale and prolapsing in the right atrium.

Video 3: Follow-up echocardiography (long axis view) three weeks later showing a decrease in thrombus size.

Video 4: Follow-up echocardiography (apical 5-chamber view) three weeks later showing a decrease in thrombus size. 


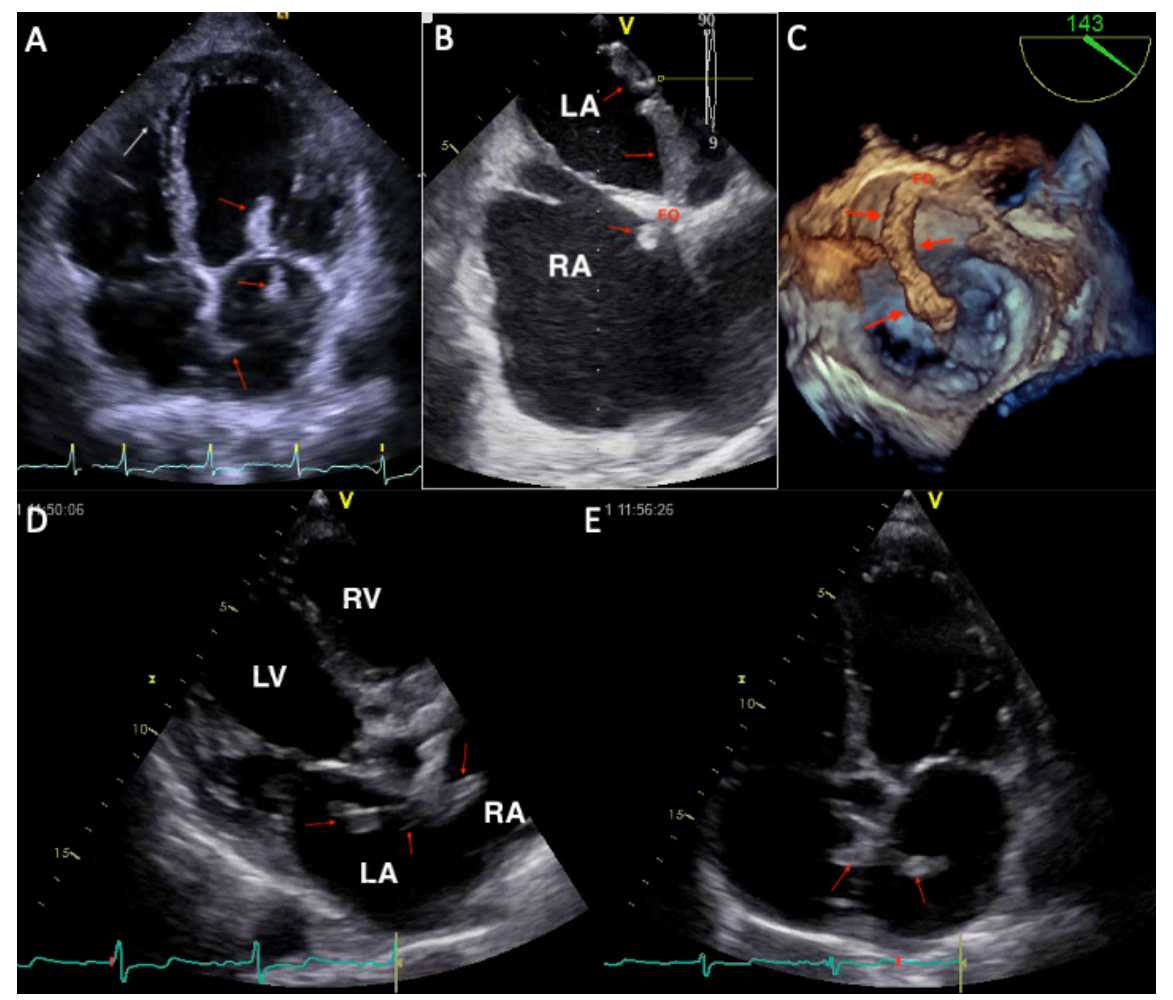

